\title{
Real-Time Analysis of Alarm Pheromone Emission by the Pea Aphid (Acyrthosiphon Pisum) Under Predation
}

\author{
Ezra G. Schwartzberg • Grit Kunert • Claudia Stephan • \\ Anja David • Ursula S. R. Röse • \\ Jonathan Gershenzon • Wilhelm Boland • \\ Wolfgang W. Weisser
}

Received: 31 May 2007 /Revised: 11 October 2007 / Accepted: 22 October 2007 / Published online: 19 December 2007

(C) Springer Science + Business Media, LLC 2007

\begin{abstract}
Upon attack by predators or parasitoids, aphids emit volatile chemical alarm signals that warn other aphids of a potential risk of predation. Release rate of the major constituent of the alarm pheromone in pea aphids (Acyrthosiphon pisum), $(E)$ - $\beta$-farnesene (EBF), was measured for all nymphal and the adult stage as aphids were attacked individually by lacewing (Chrysoperla carnae) larvae. Volatilization of EBF from aphids under attack was quantified continuously for $60 \mathrm{~min}$ at 2-min intervals with a rapid gas chromatography technique ( $\mathrm{zNose}^{\mathrm{TM}}$ ) to monitor headspace emissions. After an initial burst, EBF volatilization declined exponentially, and detectable amounts were still present after $30 \mathrm{~min}$ in most cases. Total emission of EBF averaged $16.33 \pm 1.54 \mathrm{ng}$ and ranged from 1.18 to $48.85 \mathrm{ng}$. Emission was higher in
\end{abstract}

E. G. Schwartzberg • G. Kunert • A. David • U. S. R. Röse •

J. Gershenzon $\cdot$ W. Boland

Max Planck Institute for Chemical Ecology,

Hans-Knöll-Straße 8,

07745 Jena, Germany

Present address:

E. G. Schwartzberg $(\triangle)$

Department of Entomology, Chemical Ecology Laboratory,

The Pennsylvania State University,

University Park, PA 16802, USA

e-mail: egs10@psu.edu

G. Kunert $\cdot$ C. Stephan $\cdot$ W. W. Weisser

Institute of Ecology, Friedrich-Schiller University,

Dornburger Str. 159,

07743 Jena, Germany

Present address:

U. S. R. Röse

ICG-III, Phytosphere, Research Center Juelich,

52425 Juelich, Germany nymphs as compared to adults. No differences between pea aphid life stages were detected for their speed of alarm signal emission in response to lacewing larvae attack. This is the first time that alarm pheromone emission from single aphids has been reported.

Keywords Aphid alarm pheromone $\cdot(E)-\beta$-farnesene .

Pea aphid $\cdot$ Acyrthosiphon pisum $\cdot$ zNose

\section{Introduction}

Insects possess a wide range of chemical defenses that aid in protection against predation. Aphids are soft-bodied insects and are especially prone to attack by predators and parasitoids (Dixon 1998). Whereas aphids have means of protection against natural enemies through physical adaptations such as wax production (Smith 1999) and sclerotization among soldier forms of social aphids (Wool 2004), their primary protection from predators and parasitoids consists of escape responses mediated by the use of alarm pheromone signaling (Pickett et al. 1992; Dixon 1998). Aphids emit alarm pheromone by secreting a droplet of liquid from the ends of the siphunculi that contain chemical compounds. These compounds volatilize from the surface of the cornicle droplet and induce defense-related behavioral changes in conspecifics. Specifically, when aphids secrete a cornicle droplet in response to an attack by a predator or parasitoid, or in response to an artificial probe to simulate an attack, nearby aphids move away from the area and will often drop from their host plants (Losey and Denno 1998a; Braendle and Weisser 2001). Aphids have also been shown to mark predators with alarm pheromone by daubing them with cornicle droplets. This acts as a traveling signal, causing 
aphids to disperse in response to an approaching predator (Mondor and Roitberg 2004). Recent results suggest that groups of aphids exposed to synthetic (E)- $\beta$-farnesene (EBF), the main component of aphid alarm pheromone, (Bowers et al. 1972; Pickett and Griffiths 1980; Francis et al. 2005a), will be more likely to produce winged offspring (Kunert et al. 2005; Podjasek et al. 2005).

In addition to inducing defense-related behavioral changes in conspecifics, aphid alarm pheromone also induces behavioral changes in the predators and parasitoids that exploit them, acting as a kairomone used to home in on their aphid prey (Nakumuta 1991; Boo et al. 1998; Powell and Pickett 2003; Francis et al. 2004; Francis et al. 2005b).

Although the alarm pheromone, EBF, is used by many aphids of the family Aphididae, some species are more responsive to alarm pheromone than others (Losey and Denno 1998b), with variation in response to an alarm signal even among different clonal lines of the same species (Muller 1983; Braendle and Weisser 2001). In the pea aphid, Acyrthosiphon pisum, there is little variation in the amount of EBF emitted from different clonal lines in response to predation (Schwartzberg et al., unpublished); however, it has been shown that the concentration of EBF within cornicle droplets and the propensity to produce a cornicle droplet in response to an attack varies among instars (Mondor et al. 2000).

A number of studies have attempted to quantify the amounts of alarm pheromone produced by single aphids. Mondor et al. (2000) measured the EBF content within cornicle secretions of aphids disturbed by using a blunt probe. Schwartzberg et al. (unpublished) measured EBF in the headspace of aphid colonies attacked by predators, and the amount of EBF released by crushed aphids was measured by Francis et al. (2004) and Byers (2005). So far, however, no study has taken into consideration how natural predation may affect EBF emission from individual aphids, and how this may differ among aphid instars. Because the emission of EBF from a cornicle droplet is likely to be influenced by (1) the size and surface area of the cornicle droplet produced, (2) whether it is secreted as a single event, or continuously over a period of time, and (3) the concentration of EBF within the droplet, measuring the actual emission from single aphids is a much more direct and accurate method for comparing alarm signal among instars.

In this paper, we use a $\mathrm{zNose}^{\mathrm{TM}}$ (Electronic Sensor Technology, Newbury Park, CA, USA), which is an instrument for rapid gas chromatography that is capable of repeated quantitative sampling of headspace volatiles (Kunert et al. 2002; Tholl et al. 2006) to ask the following questions: (1) What is the temporal pattern of EBF volatization after attack by a predator? (2) Is there a time-lag between attack by the predator and EBF emission? (3) Do different instars differ in the amount of EBF emitted?

\section{Materials and Methods}

Pea aphids, Acyrthosiphon pisum (Hemiptera: Aphididae), used for alarm pheromone quantification were obtained from a naturally occurring pink pea aphid colony, "Jena Pink 1" (JP1), and were reared on 2-week-old dwarf broad bean plants, Vicia faba L. (The Sutton; Nickerson-Zwaan, UK) in $10-\mathrm{cm}$ plastic pots with $16 \mathrm{~h}$ light: $8 \mathrm{~h}$ dark, $20^{\circ} \mathrm{C}$, $75 \%$ relative humidity. Lacewing larvae, Chrysoperla carnea (Neuroptera: Chrysopidae), were obtained from a commercial supplier (Katz Biotech Services, Welzheim, Germany), and larvae were maintained on bean plants infested with pea aphids until they reached the second instar.

Aphid alarm pheromone emission was monitored from members of all instars, including the adult stage, by using a zNose $^{\mathrm{TM}}$. Air was sampled from individual aphids and lacewing larvae within air entrainment vials (Fig. 1). The stainless steel inlet needle of the $\mathrm{zNose}^{\mathrm{TM}}$ was inserted through a small hole in the septum of a 4-ml glass vial. A clean air inlet was created by inserting a hypodermic syringe tip into the septum adjacent to the $\mathrm{zNose} \mathrm{TM}^{\mathrm{TM}}$ inlet needle.

The $z N_{o s e}{ }^{T M}$ was programmed to sample air within the vial every 2 min with a flow rate of $30 \mathrm{ml} \mathrm{min}^{-1}$ for $20 \mathrm{~s}$, drawing a total of $10 \mathrm{ml}$ of air through the preconcentration trap during each $20 \mathrm{~s}$ sampling period. This quantity of air allowed the entrainment vial to be purged of most airborne volatiles prior to subsequent samples. The air was sampled repeatedly from the entrainment vial every $2 \mathrm{~min}$ for the duration of the bioassay.

For each replicate, a single aphid was introduced into a clean vial immediately after a baseline air entrainment. Once the aphid headspace was sampled, one second instar lacewing was introduced into the chamber with the aphid. Lacewing larvae did not attack aphids immediately so we were able to collect headspace volatile samples from aphid and lacewing larva before attack. Air within the vial was collected every 2 min from the introduction of the lacewing larva until 60 min after the beginning of lacewing attack (determined visually). Lacewing larvae feed by piercing their mouthparts into the soft bodies of their prey. The exoskeletons of prey are not consumed, and remain present even after feeding has finished. Therefore, it was not possible to discern exactly when the lacewing had stopped feeding. Because aphid alarm pheromone signals can exist beyond the life of an emitting aphid, the alarm signal was recorded even after the interior of the aphid was apparently fully consumed and the aphid was dead. We determined in preliminary tests that $60 \mathrm{~min}$ was a sufficient time period to measure all of the emitted alarm pheromone from an attacked aphid.

Air drawn from vials was collected on a Tenax ${ }^{\mathrm{TM}}$ absorbent trap during sampling. Trapped compounds were desorbed from the Tenax ${ }^{\mathrm{TM}}$ trap $\left(300^{\circ} \mathrm{C}\right.$ for $\left.30 \mathrm{~ms}\right)$ and

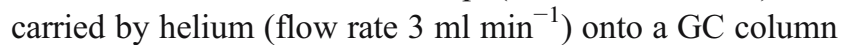


Fig. 1 Air entrainment setup showing $4 \mathrm{ml}$ vial with inserted ZNose ${ }^{\mathrm{TM}}$ sampling needle and air inlet needle

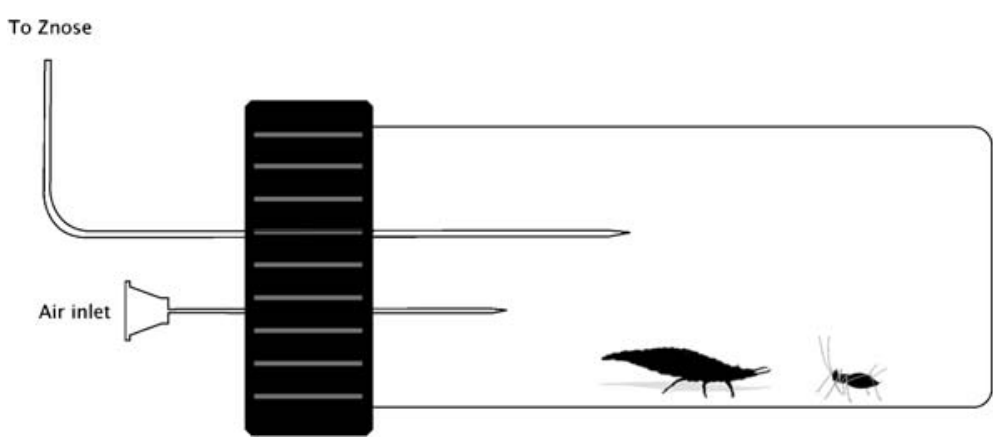

( $1 \mathrm{~m}, \mathrm{DB}-5$, film thickness $0.25 \mu \mathrm{m}$, ID $0.25 \mathrm{~mm}$ ), which was programmed from 40 to $180^{\circ} \mathrm{C}$ at $5^{\circ} \mathrm{C} \mathrm{s}^{-1}$. As compounds exited the column, they were quantified as they pass over a temperature-controlled sensitive surface acoustic wave (SAW) quartz microbalance detector set at $40^{\circ} \mathrm{C}$. The resonance frequency $[\mathrm{Hz}]$ of the SAW detector changes in a linear fashion as compounds from the column are attached to and heated off of the quartz detector. For quantification purposes, response factors were calculated by comparison with synthetic EBF (Bedoukian, Danbury, CT, USA) exposed to the $\mathrm{zNose}^{\mathrm{TM}}$ in known concentrations by using a heated desorber tube (glass, temperature controlled; $190^{\circ} \mathrm{C} ; 3100$ Vapor Calibrator; Electronic Sensor Technology, Newbury Park, CA, USA).

Alarm pheromone emission was quantified for nymphal stages L1 to L4 and for adult aphids ( $N=10$ for each instar). The following variables were calculated from the data and used for the analysis: (1) total emission (calculated as the sum of all recordings after attack), (2) peak emission (the highest reading after attack), and (3) the lag time between initial attack and maximum emission (calculated as the time of attack subtracted from the time of maximum emission).

Because data were not normally distributed, we used a Kruskal-Wallis test for among-instar statistical comparisons.

\section{Results}

(E)-ß-farnesene was identified based on retention time comparison to a synthetic standard. There was only one peak detected in samples from JP1-aphids, and this peak was not present in control samples without aphids. Previous collections of volatiles from entire JP1-aphid colonies and analysis by GC-MS identified this peak as EBF (Schwarzberg et al., unpublished). The calibration curve for the SAW detector response to EBF was linear $(y=0.230+0.000255 x ; y=\mathrm{ng}$ of EBF, $x=$ SAW detector response in hertz).

EBF volatization after an attack showed a typical pattern: after an initial burst it declined exponentially (Fig. 2). For the 46 cases where emission continued for more than $30 \mathrm{~min}$,
Fig. 2 Typical time course of (E)- $\beta$-farnesene emission in the experiment. This example is from a first instar aphid (L1) attacked by a lacewing larva




amounts of over $0.3 \mathrm{ng}$ per 2 min interval were measured in 30 replicates.

Total EBF emission over the entire 60 min collection period averaged $16.33 \pm 1.54 \mathrm{ng}$ and ranged from 1.18 and $48.85 \mathrm{ng}$. Emission was different between first and second instar nymphs (L1 and L2) as compared to adults $\left(\chi^{2}=\right.$ 13.34, $d f=4, P=0.01)$ with adult aphids emitting the least amount of EBF over the entire collection period (Fig. 3).

Peak EBF emission, measured as the maximum EBF emission for a 2 min interval within the total $60 \mathrm{~min}$ sampling period, had a mean of $1.98 \pm 0.32 \mathrm{ng}$ per $2 \mathrm{~min}$ and ranged from 0.12 to $16.26 \mathrm{ng}$ per $2 \mathrm{~min}$. Mean peak emission varied among instars $\left(\chi^{2}=14.34, d f=4, P=0.006\right.$, Fig. 4). While there was no significant difference in peak EBF emission among instars, adult peak emission was significantly lower.

The time lag between onset of attack by a lacewing larva and peak EBF emission was calculated to determine if different instars vary in their speed of alarm signal production. It is interesting to note that mean lag time was $10.7 \pm$ $1.7 \mathrm{~min}$ and ranged from 2 to $50 \mathrm{~min}(N=48)$. Even if outliers (time-lag $>13 \mathrm{~min}$ ) were excluded, the mean was still $5.1 \pm$ $0.4 \mathrm{~min}(N=36)$. Inspection of the 12 values with a time lag of 14 or more minutes revealed that in 10 cases (one for L1, five for L3, one for L4, and two for adults) there was no real burst of emission but rather a slow build up, associated with a low maximum emission value. In the other two cases (one L1, one L4), the observational determination of time of attack may have been wrong or there was a second burst of emission, possibly associated with a second attack by the predator. As a consequence of the variability, there was no statistical difference among instars $\left(\chi^{2}=3.98, d f=4, P=0.409\right.$, Kruskal-Wallis, Fig. 5).

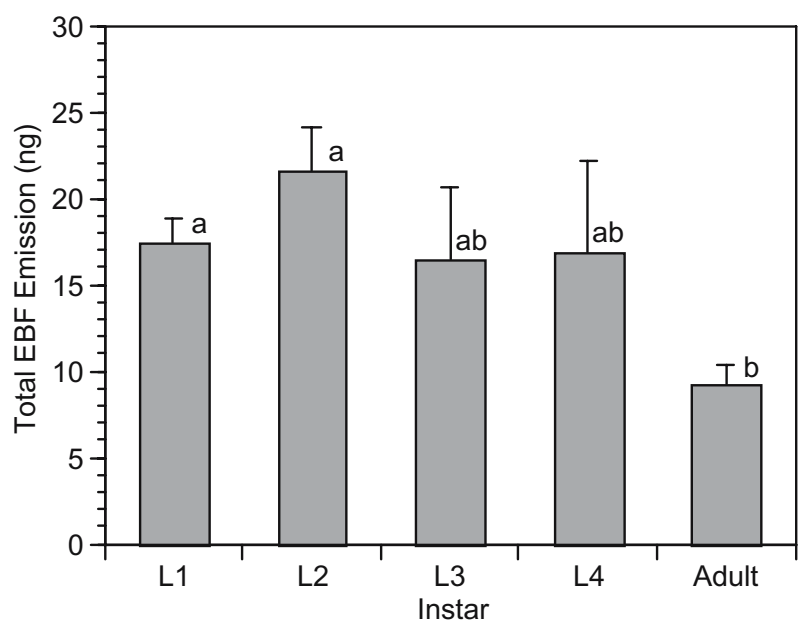

Fig. 3 Sum of $(E)-\beta$-farnesene in nanogram $\pm S E$ emitted over the entire 60-min collection period for immature instars (L1-L4) and adults. Significant differences in $(E)-\beta$-farnesene production between instars are indicated by different letters; $P \leq 0.05$, Kruskal-Wallis test, $N=10$

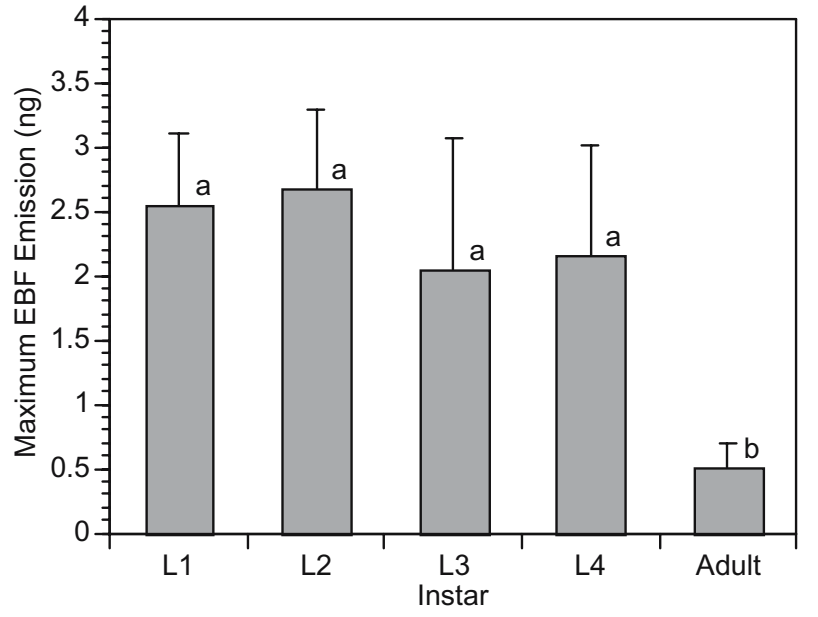

Fig. 4 Peak emission of $(E)-\beta$-farnesene in nanogram \pm SE per 2 min sampling interval. Significant differences in $(E)-\beta$-farnesene emission between instars are indicated by different letters; $P \leq 0.05$, KruskalWallis test, $N=10$

\section{Discussion}

All life stages of pea aphids can be stimulated to produce a cornicle droplet when prodded, but adults do so less consistently (Mondor et al. 2000). This was also reflected in our results that show both total EBF emission and peak emission were lower in adults than in the younger instars. In addition to the differences among instars, our analysis showed that variability of EBF emission within instars was high and that detectable amounts of alarm pheromone were still present in the headspace of aphids for a considerable time after the initiation of attack.

The $\mathrm{zNose} \mathrm{TM}^{\mathrm{TM}}$ was able to illustrate the temporal patterns of alarm pheromone emission from individual aphids. In addition to total emission over a 60 -min time period, we were

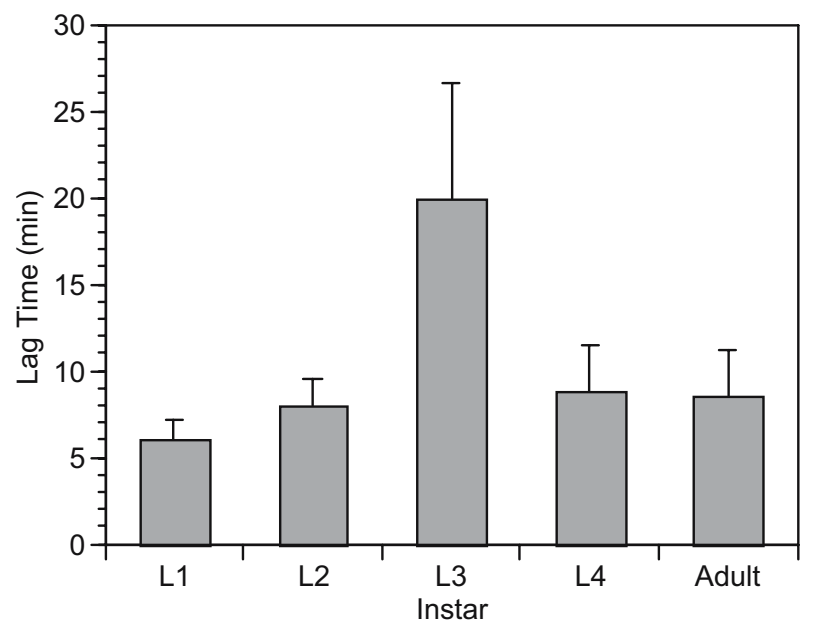

Fig. 5 Lag time between initial attack by lacewing larvae and peak emission of $(E)-\beta$-farnesene in minutes $\pm \mathrm{SE}$. There was no statistical difference in lag time among instars; $P>0.05$, Kruskal-Wallis test, $N=10$ 
able to record the initial peak of alarm signal production and latency of the alarm signal at 2 min intervals. The initial burst of alarm pheromone emission was relatively consistent among nymphal instars, but much lower in adults. The initial burst of alarm signal production is likely the most important, because aphids react within the first few seconds of perceiving the signal. Nevertheless, there was variability in peak emission from less than $1 \mathrm{ng}$ up to $16 \mathrm{ng}$ emitted in a single 2-min interval. Whereas we were able to determine visually when a lacewing larva grabbed an aphid, it was not possible to visually rate the strength of the attack and the time when the aphid died. The variability in peak emission may, therefore, reflect both the strength of initial attack and individual variability in cornicle secretion.

Aphid predators differ greatly in their handling time of prey, depending on their mode of feeding and the relative size of the predator compared to the prey. While we were not able to determine exactly the feeding time of the lacewing larvae in the vials, results from a separate experiment showed that feeding time of similar-sized lacewing larvae was 35.6 \pm $4.8 \mathrm{~min}$ min after attack for adult pea aphids $(N=12$ larvae; Weisser, unpublished). In contrast, adult ladybirds consume an adult pea aphid within a few seconds. Handling times of ladybird larvae or hoverfly larvae are likely to be intermediate. Although most of the EBF that aphids produced during a lacewing larva attack volatilized during the first $15 \mathrm{~min}$ in our closed system, they continued to emit measurable quantities for $60 \mathrm{~min}$. It is not known how EBF is synthesized, stored, and released by aphids during predation events, and it is possible that the larvae continually replenished their cornicle droplets while they were still not entirely eaten. Whereas our collection method was limited to a confined space with limited natural airflow in contrast to an open, natural system, where air currents may result in a faster rate of volatile emission from cornicle droplets, we believe that the EBF volatilization dynamics observed reflect natural dynamics. In experiments with adult ladybirds, a single flush of air after predation was in almost all cases enough to capture the entire EBF released (Hatano, personal communication). Regardless of whether natural dynamics may be slightly different, our design allowed for nonbiased comparison of signal strength and duration between nymphal and adult instars. It is interesting to note that these two temporally different signals were quantitatively similar among all nymphal instars.

Our results are consistent with those of other studies that attempt to measure alarm pheromone production from multiple life stages of aphids (Mondor et al. 2000; Byers 2005). In an elegant study, Mondor et al. (2000) determined the amounts of EBF in cornicle droplets after manual stimulation. Second to fourth instars contained on average between 11 and $13 \mathrm{ng}$, about five times higher than average peak emission in our study and slightly less than the 16 to
$21 \mathrm{ng}$ we found to be released over $60 \mathrm{~min}$. In adults, Mondor et al. (2000) found on average 3-4 ng EBF in a cornicle droplet, about half of what we measured over the 60-min period, whereas EBF in adult cornicle droplets measured by Mondor et al. (2000) was four times as high as the mean peak emission in our study. The major difference between our and Mondor et al. (2000) results was for first instars, which emitted about $2 \mathrm{ng}$ in their study, in contrast to $17 \mathrm{ng}$ in ours. It is interesting to note that the $2 \mathrm{ng}$ corresponded to what we measured as peak emission in our study. As mentioned above, it is conceivable that as the predator continues to feed on the aphid more EBF is released, either from a reservoir or by de novo synthesis, which would explain the higher amounts detected in summed headspace collections in our study. However, one thing to consider is that different predators feed differently, and this may have an effect on the duration and strength of the signal. For instance, a larger predator that completely consumes its prey may effectively quench the alarm signal of an aphid by consuming it.

Production of alarm pheromone, although altruistic in principle, is thought to be adaptive among groups of closely related individuals such as clones within aphid colonies because it allows related individuals to disperse and avoid predation or parasitization. However, alarm pheromone production comes at a cost (Mondor and Roitberg 2003; Byers 2005). This cost can be in terms of reproduction (Mondor and Roitberg 2003) or through the attraction of natural enemies (e.g., Francis et al. 2005a). Little is known about the emission dynamics of EBF in aphid colonies and how aphids may adjust alarm pheromone release to the actual ecological situation. An understanding of the temporal dynamics of EBF is necessary to further elucidate the role of aphid alarm pheromone in aphid-natural enemy interactions.

Acknowledgements The authors thank Katz Biotech Services for supplying C. carnea free of charge and Michael J. Domingue for comments on earlier drafts of this manuscript. This project was supported by the International Max Planck Research School, Shortterm Research Fellowship. E.G.S. is grateful to both the Max Planck Institute for Chemical Ecology and Friedrich-Schiller University for providing facilities to conduct this research as well as members of the Weisser and Gershenzon labs for their hospitality.

\section{References}

Boo, K. S., Chung, I. B., Han K. S., Pickett, J. A., and Wadhams, L. J. 1998. Response of the lacewing Chrysopa cognata to pheromones of its aphid prey. J. Chem. Ecol. 24:631-643.

Bowers, W. S., WebB, R. E., NAult, L. R., and DutKy S. R. 1972. Aphid alarm pheromone: isolation, identification, synthesis. Science 177:1121-1122.

Braendle, C., and Weisser, W. W. 2001. Variation in escape behavior of red and green clones of the pea aphid. J. Insect Behav. 14:497-509. 
BYERS, J. A. 2005. A cost of alarm pheromone production in cotton aphids, Aphis gossypii. Naturwissenschaften 92:69-72.

DiXON, A. F. G. 1998. Aphid Ecology. Chapman \& Hall, London.

Francis, F., Lognay, G., and Haubruge, E. 2004. Olfactory responses to aphid and host plant volatile releases: (E)-betafarnesene, an effective kairomone for the predator Adalia bipunctata. J. Chem. Ecol. 30:741-755.

Francis, F., VANDERMoten, S., VerhegGen, F., Lognay, G., and HAUBRUGE, E. 2005a. Is (E)-beta-farnesene the only volatile terpenoid in aphids? J. Appl. Entomol. 129:6-11.

Francis, F. D., Martin, T., Lognay, G., and Haubruge, E. 2005b. Role of (E)-beta-farnesene in systematic aphid prey location by Episyrphus balteatus larvae (Diptera : Syrphidae). Eur. J. Entomol. 102:431-436.

Kunert, G., Otto, S., Rose, U. S. R., GershenZon, J., and Weisser, W. W. 2005. Alarm pheromone mediates production of winged dispersal morphs in aphids. Ecol. Lett. 8:596-603.

Kunert, M., BiedermanN, A., Koch, T., and Boland, W. 2002. Ultrafast sampling and analysis of plant volatiles by a hand-held miniaturised GC with pre-concentration unit: kinetic and quantitative aspects of plant volatile production. J. Sep. Sci. 25:677684.

LOSEY, J. E. and DENNO, R. F. 1998a. The escape response of pea aphids to foliar-foraging predators: factors affecting dropping behaviour. Ecol. Entomol. 23:53-61.

LOSEY, J. E. and DENNO, R. F. 1998b. Interspecific variation in the escape responses of aphids: effect on risk of predation from foliar-foraging and ground-foraging predators. Oecologia 115: $245-252$.

Mondor, E. B., and RoitberG, B. D. 2003. Age-dependent fitness costs of alarm signaling in aphids. Can. J. Zool. 81:757-762.
Mondor, E. B., and RoItBerg, B. D. 2004. Inclusive fitmess benefits of scent-marking predators. Proc. R. Soc. Lond. B 271:341-343.

Mondor, E. B., Baird, D. S., Slessor, K. N., and RoItberG, B. D. 2000. Ontogeny of alarm pheromone secretion in pea aphid, Acyrthosiphon pisum. J. Chem. Ecol. 26:2875-2882.

MULLER, F. P. 1983. Differential alarm pheromone responses between strains of the aphid Acyrthosiphon pisum. Entomol. Exp. Appl. 34:347-348.

NAKUMUTA, K. 1991. Aphid alarm pheromone component, (E)-betafarnesene, and local search by a predatory lady beetle, Coccinella septempunctata bruckii Mulsant (Coleoptera: Coccinellidae). Appl. Entomol. Zool. 26:1-7.

PicketT, J. A., and GRIFFITHS, D. C. 1980. Composition of aphid alarm pheromones. J. Chem. Ecol. 6:349-360.

PicketT, J. A., Wadhams, L. J., Woodcock, C. M., and Hardie J. 1992. The chemical ecology of aphids. Annu. Rev. Entomol. 37:67-90.

PodjaseK, J. O., BosnJaK, L. M., BRoOKer, D. J., and Mondor, E. B. 2005. Alarm pheromone induces a transgenerational wing polyphenism in the pea aphid, Acyrthosiphon pisum. Can. J. Zool. 83:1138-1141.

Powell, W. and PiCKETT, J. A. 2003. Manipulation of parasitoids for aphid pest management: progress and prospects. Pest Manag. Sci. 59:149-155.

SMITH, R. G. 1999. Wax glands, wax production and the functional significance of wax use in three aphid species (Homoptera: Aphididae). J. Nat. Hist. 33:513-530.

Tholl, D., Boland, W., Hansel, A., Loreto, F., Rose, U.S.R., and SCHNITZLER, J. 2006. Practical approaches to plant volatile analysis. Plant J. 45:540-560.

WooL, D. 2004. Galling aphids: specialization, biological complexity, and variation. Annu. Rev. Entomol. 49:175-192. 